\begin{tabular}{|c|l|}
\hline Title & Multi-objective optimization of curvilinear fiber shapes for laminated composite plates by using NSGA-II \\
\hline Author(s) & Honda, Shinya; Igarashi, Teruki; Narita, Y oshihiro \\
\hline Citation & $\begin{array}{l}\text { Composites Part B: Engineering, 45(1), 1071-1078 } \\
\text { https://doi.org/10.10161.Compositesb.2012.07.056 }\end{array}$ \\
\hline Issue Date & 2013-02 \\
\hline Doc URL & http://hdl.handle.net/2115/52181 \\
\hline Type & article(author version) \\
\hline File Information & CBE45-1_1071-1078.pdf \\
\hline
\end{tabular}

Instructions for use 


\title{
Multi-Objective Optimization of Curvilinear Fiber Shapes for Laminated Composite Plates by Using NSGA-II
}

$\underline{\text { Shinya Honda }}^{*}$, Teruki Igarashi, and Yoshihiro Narita

Faculty of Engineering, Hokkaido University, N13W8, Kita-ku, Sapporo, Hokkaido, 060-8628, Japan

*Corresponding author, Email: honda@eng.hokudai.ac.jp

Tel: +81-11-706-6415, Fax: +81-11-706-6416

\begin{abstract}
A multi-objective optimization approach for fibrous composite plates with curvilinear fibers is proposed in the present study. The non-dominated sorting genetic algorithm (NSGA-II) is used to obtain Pareto-optimum solutions, where the presence of a trade-off relation between the mechanical performance and curvatures of reinforcing fibers has been established. The in-plane strength around a circular hole in a finite-width plate with Tsai-Wu failure criteria or the fundamental frequency of the square plate is the mechanical objective function, while the average value of fiber curvatures is used as the conflicting objective function. Numerical results were obtained for angle-ply plates and the effectiveness of the present design approach is demonstrated by widely distributed Pareto-optimum solutions.
\end{abstract}

\section{Keywords:}
A. Plates
B. Strength
B. Vibration
C. Finite element analysis (FEA) 


\section{Introduction}

The present study proposes a multi-objective optimization for the fibrous composite plates with curvilinear fibers. Composites of this kind became feasible using an innovative method recently developed to produce composites with curvilinear fibers. Details of the manufacturing method of composites with curvilinear fibers are written in [1]. As expected, the mechanical properties of composite plates reinforced by curvilinear fibers strongly depend on the fiber orientations, and such composites display non-uniform stiffness and anisotropy. Compared with homogenously anisotropic plates with straight fibers, this offers the potential for designing task-specific fibrous composite plates using curvilinear fibers.

Local anisotropy is found in many natural compounds and such compounds quite often show properties superior to homogenous engineering structures. One example is the naturally occurring holes, foramina, in bone that do not display crack initiation or growth since the HAp (hydroxyapatite) crystals are tangentially oriented around the foramen edges [2]. In this way, natural compounds have locally anisotropic properties distributed close to optimal and this makes the structural performance more effective than homogenous compounds.

In the previous work [3], the authors have shown advantages of curvilinear fibers. The obtained plates with curvilinear fibers result in a higher fundamental frequency than plates with straight fibers, while some optimized plates indicate optimum fiber shapes with very large curvatures which may be impractical to fabricate. Based on these findings where a trade-off relation exists between the mechanical properties and the geometrical features of the curved fibers, the present work extends the previous work to a multi-objective optimization, dealing with two conflicting objectives. One is the mechanical value where in-plane strength around a 
circular hole within the finitely wide plate is employed as well as the natural frequency of square plates. The other objective is the practicality of the fiber shapes that is expressed as an average value of the fiber curvatures defined at the center of elements in the finite element analysis.

There have been several reports on locally anisotropic structures. Martin and Leissa [4] first proposed a variable stiffness concept by varying fiber spacings to improve the vibration and buckling performance of plates with the Ritz method. Hyer and Lee [5] employed the finite element analysis (FEA) to analyze the buckling performance of plates with variable stiffness formed by curvilinear fibers. As for manufacturing, Wu et al. [6] first reported feasibility of composite plates with curvilinear fibers where responses of compression forces were investigated analytically and experimentally. Gürdal et al. [1,7-11] defined fiber shapes by the linear changes in fiber orientation angles to require locally desired material properties for plates and conical shells, and confirmed that such structures have superior mechanical properties compared with homogenous plates by research including experimental and analytical tests. The authors $[3,12]$ have calculated the natural frequencies of plates reinforced by arbitrarily shaped fibers using the Ritz method, and showed that plates with local properties have advantages over homogenously anisotropic plates with straight fibers in terms of vibration properties.

For optimization of locally anisotropic composites, Setoodeh et al. [13-15] studied the optimization of plates with local properties by employing lamination parameters which describe laminated plate properties in simple form as design variables. Van Campen et al., recently, proposed a method for generating fiber shapes [16]. The authors here also used lamination parameters to design optimum short fiber distributions [17]. Blom et al. [18, 19] designed conical shells using curvilinear fibers for maximizing fundamental frequencies while 
imposing manufacturing constraints, and optimized fiber paths using stream line analogy. Cho and Rowlands [20] and Huang and Haftka [21] designed fiber directions to reduce stress concentrations around circular holes. Parnas et al. [22] applied curvilinear fibers to a minimum weight design.

There are also studies using multi-objective approaches to composite structures [23-25]. Up to the present, however, no paper has revealed trade-off relationship between fiber shapes and mechanical properties for the composite with curvilinear fibers defined by the polynomial surface. In this study, the objective functions of this optimization are mechanical properties: a Tsai-Wu failure index and natural frequency, and an average value of fiber curvatures. The design variables are the coefficients of a cubic polynomial function. The curvilinear fiber shapes are expressed by the projections of contour lines of cubic polynomial surfaces. A non-dominated sorting genetic algorithm method with a crowded distance metric (NSGA-II) [26] is used for searching Pareto-optimum solutions. An originally developed finite element analysis code is employed with an iso-parametric eight-node element $[27,28]$ to calculate the mechanical properties. The numerical results showed that the present multi-objective optimization technique is able to determine the widely distributed Pareto-optimum solutions. 


\section{Analysis and Optimization method}

\subsection{Expression of curvilinear fiber shape}

A cubic polynomial function $f(x, y)$ is introduced to define curvilinearly shaped fibers, as

$$
f(x, y)=c_{00}+c_{10} x+c_{01} y+c_{20} x^{2}+c_{11} x y+c_{02} y^{2}+c_{30} x^{3}+c_{21} x^{2} y+c_{12} x y^{2}+c_{03} y^{3}
$$

where $c_{i j}(i, j=0,1,2,3)$ are shape coefficients which determine the surface shape. An example of a surface and the corresponding curves are shown in Fig. 1(a), and Fig. 1(b) indicates contour lines of the surface projected on the horizontal plane. A range of shapes of surfaces could be described with this expression by varying the values of the coefficients $c_{i j}$. It is difficult to employ continuously curved fibers in the FEA, and the fibers are discritized at each element and dealt with as straight fibers. The fiber orientation angle $\theta_{n, p}$ at the $p$ th element in the $n$th layer is defined as the tangential direction of the contour lines using the coordinates $\left(x_{c}, y_{c}\right)_{p}$ of the center of the element, which is calculated using the following equation.

$$
\theta_{n, p}\left(x_{c}, y_{c}\right)=\left.\tan ^{-1}\left(-\frac{\partial f / \partial x}{\partial f / \partial y}\right)\right|_{x=x_{c}, y=y_{c}}
$$

It is assumed that each element has straight fibers with a constant fiber volume fraction but different orientation angle, and the discrete shape of Fig. 1(b) is shown in Fig. 1(c). The angles are defined by a continuous polynomial function and this description imposes continuity constraints on the fiber directions. Further, the present description is more effective and flexible than using the spline function [12] since there is no requirement for solving simultaneous equations to determine the fiber shapes and it simply accepts multi-valued functions.

The curvature of a surface defined by Eq. (1) at an arbitrary point $\left(x^{*}, y^{*}\right)$ is expressed as

$$
\kappa\left(x^{*}, y^{*}\right)=\frac{1}{\left(f_{x}^{2}+f_{y}^{2}\right)^{3 / 2}}\left[\begin{array}{ll}
-f_{y} & f_{x}
\end{array}\right]\left[\begin{array}{ll}
f_{x x} & f_{x y} \\
f_{x y} & f_{y y}
\end{array}\right]\left[\begin{array}{c}
-f_{y} \\
f_{x}
\end{array}\right]
$$


where the subscripts stand for values of partial differentials at a point $\left(x^{*}, y^{*}\right)$ with respect to $x$ and $y$, for example $f_{x}$ and $f_{y y}$ are $\partial f / \partial x$ and $\partial^{2} f l(\partial y)^{2}$, respectively. By using Eq. (3), the average value of the curvatures within the plate, which is one of the objective functions in this optimization, is calculated by

$$
\bar{\kappa}=\frac{1}{n_{e}} \sum_{k=1}^{n_{e}} \kappa\left(x_{c}, y_{c}\right)^{(k)}
$$

where $n_{e}$ is the number of elements in the area of evaluation, and $\left(x_{c}, y_{c}\right)^{(k)}$ is the coordinates at the center of the element for the $k$ th element. In this way, the present method evaluates curvature value at discrete points and these points do not necessarily correspond to the maximum curvature point. Hence, the averaged curvature is chosen as an objective function $\underline{\text { here. }}$

For obtaining widely distributed Pareto-optimum front, the present study added no constraints on the fiber curvatures, resulting in impractical fiber shapes with excessive curvatures. The value of $\overline{\bar{\kappa}}$ can be used as an index of practicality of fiber shape. As the value of $\underline{\bar{\kappa}}$ becomes small, the fiber shapes become straight, and can be regarded as a fiber shape with $\underline{\text { inferior mechanical properties compared with higher curvatures. }}$

\subsection{Multi-objective optimization}

A multi-objective genetic algorithm method based on the non-dominated sorting genetic algorithm (NSGA-II) proposed by Deb [26] is used to obtain Pareto-optimal solutions in the present paper. The NSGA-II is implemented with an effective sorting method based on individual ranking by non-dominated sorting and a crowded distance metric sorting which evaluates the population density of solutions in the same rank. It is known that the NSGA-II has a good search performance for widely distributed Pareto-optimal solutions with conflicting 
objectives.

Figure 2 shows a schematic representation of the non-dominated sorting in an objective function field. Individuals such as $x^{(1)}, x^{(4)}, x^{(6)}$, and $x^{(7)}$ are assigned ranks as rank 1 since there is no individual superior to them with respect to $f_{1}(x)$ and $f_{2}(x)$ when the minimizing problem of both conflicting objectives are considered. After elimination of individuals classified as rank 1, individuals with rank 2 are selected, and this process is repeated until all individuals are classified.

An example of the crowded distance metric is indicated in Fig. 3, where the density of population is evaluated for individuals in the same rank, with distances $\Delta d_{1}$ and $\Delta d_{2}$ between an individual $x^{(i)}$ and individuals $x^{(i-1)}$ and $x^{(i+1)}$ in the $f_{1}$ and $f_{2}$ directions. The process evaluates the sum of $\Delta d_{1}$ and $\Delta d_{2}$ for each individual, and the one with the larger distance is ascribed as valuable since such individuals contribute to maintain the diversity of the solutions. Individuals located at both edges of the same rank such as $x^{(1)}$ and $x^{(n)}$ are also important to obtain widely distributed Pareto-optimal solutions, and large distances are assigned to these and the hundredfold value of the sum of $l_{1}$ and $l_{2}$ is used as the distances for individuals at both edges in this study.

Figure 4 shows the flow chart of NSGA-II and each process is summarized as follows.

(1) Initial parent population $P_{0}$ is generated by random numbers, and the non-dominated sorting is implemented on $P_{0}$ where each individual is ranked based on the dominance relation in the objective space.

(2) Individual within each rank is sorted again based on the crowded distance metric where the population density is evaluated. 
(3) Individuals selected by a tournament selection are stored in an intermediate mating pool which has a high probability for occurrence of better ranked and less crowded solutions.

(4) In the mating pool, genetic operations such as crossover and mutation generate the child populations $Q_{t}$ where subscript " $t$ " denotes the number of generations.

(5) An integrated population $R_{t}$ is created by combining $P_{t}$ and $Q_{t}$, and fitness values are assigned to all individuals by the non-dominated sorting and crowded distance sorting.

(6) Finally, individuals with better fitness are selected by elitist sorting and these become the parent individuals $P_{t+1}$.

(7) Steps 2 to 6 are repeated until $t=t_{\max }$.

(8) Individuals with rank 1 among parents at $P_{t \max }$ are Pareto-optimal solutions.

A termination criterion is applied by using the number of generations. If the number of generations becomes larger than the maximum number of generations $t_{\max }$, defined preliminary by the designer, the search process is terminated and the obtained individuals are considered as a set of the Pareto-optimal solutions.

The genetic operations employed here are a two point crossover with probability $p_{c}$ and a simple mutation with variable probabilities $p_{m}$, depending on the number of generation, defined by

$$
\begin{array}{ll}
p_{m}=p_{m 1}+\frac{p_{m \text { max }}-p_{m 1}}{\left(\frac{t_{\text {max }}}{2}-1\right)} \times(t-1) & \left(t \leq \frac{t_{\text {max }}}{2}\right) \\
p_{m}=p_{m \text { max }} & \left(t \leq \frac{t_{\text {max }}}{2}\right)
\end{array}
$$


where $p_{m 1}$ is the probability of mutation for the first generation and is defined as $1 /$ (length of chromosome string, $L$ ). Until half the generations, $p_{m}$ increases linearly, and then becomes a constant $p_{m \text { max }}$ after the half-way point. This is performed to keep the diversity among individuals.

\subsection{Formulation of the problem}

The present paper deals with two kinds of multi-objective optimization problems. The first problem is (i) maximizing the fundamental frequency of a square plate, and the second is (ii) minimizing the Tsai-Wu failure index $\Phi$ around a circular hole in a plate of finite width. For both problems, conflicting objective function of mechanical properties of the plate is the average curvature of fibers given by Eq. (4). Thus, the present optimization problem can be formulated as follows.

(i) Maximizing $\Omega_{1}$ and minimizing $(\bar{\kappa})_{k} \quad(k=1)$

(ii) Minimizing $(\Phi)_{k}(k=1,2)$ and $(\bar{\kappa})_{k}(k=1)$

Design variables $c_{i j}(i, j=0,1,2,3)(k=1)$

Subject to $-2.0 \leq c_{i j} \leq 2.0\left(\Delta c_{i j}=0.1\right) \quad(i, j=0,1,2$, and 3$)$

where $k=1$ and 2 are the "+layer" and "-layer". Only a symmetrically 8-layered laminated plate with $\left[(+ \text { layer/-layer })_{2}\right] \mathrm{s}$ is considered in the present paper, and "+layer" has a fiber shape defined by the optimized $c_{i j}$ and "- layer" has fiber shapes symmetric to the "+layer" with respect to the $x$ axis. It is also possible to apply the method to the plate with different lay-up conditions by assigning design variables in each layer (i.e., $k>2$ ). This causes a high calculation cost, resulting in long calculation time. To avoid this, the present study limits the laminated configuration to $\left[(+ \text { layer/-layer })_{2}\right] \mathrm{s} . \Delta c_{i j}$ is the increment of the design variable.

The objective function (i) $\Omega_{1}$ is the normalized fundamental frequency, the first frequency 
parameter, of the plate defined by

$$
\Omega=\omega a^{2} \sqrt{\frac{\rho h}{D_{0}}}
$$

where $\omega$ is the angular frequency, $a$ is the representative length of the plate, $\rho$ is the density of the plate, and $D_{0}=E_{2} h^{3} / 12\left(1-v_{12} v_{21}\right)$ is the reference stiffness; $E_{i}$ and $v_{i j}(i, j=1,2)$ are Young's modulus and Poisson's ratio in each direction with the subscripts 1 and 2 standing for the fiber and the normal to the fiber directions; $h$ is the thickness of the plate and $a / h=100$ is used. To calculate the objective function, an originally developed FEA coded with iso-parametric eight-node elements [27] based on the first order shear deformation theory (FSDT) [28] is used.

The other objective (ii) $(\Phi)_{k}(k=1,2)$ is the strength of the plate represented by the Tsai-Wu failure index. An index $(\Phi)_{k} \geq 1$ means the material failure and is minimized in this problem. With the stresses in the material principal axis directions $\left(\sigma_{1}, \sigma_{2}\right.$, and $\left.\tau_{12}\right)$ obtained by transforming $\sigma_{x}, \sigma_{y}$, and $\tau_{x y}$, the Tsai-Wu failure index is defined by

$$
\Phi=F_{1} \sigma_{1}+F_{2} \sigma_{2}+F_{11} \sigma_{1}^{2}+F_{22} \sigma_{2}^{2}+F_{12} \sigma_{1} \sigma_{2}+F_{66} \tau_{12}^{2}
$$

Where

$$
\begin{aligned}
& F_{1}=\left(X_{c}-X_{t}\right) /\left(X_{c} X_{t}\right), \quad F_{2}=\left(Y_{c}-Y_{t}\right) /\left(Y_{c} Y_{t}\right) \\
& F_{11}=1 /\left(X_{c} X_{t}\right), \quad F_{22}=1 /\left(Y_{c} Y_{t}\right), \quad F_{12}=1 /\left(X_{c} X_{t} Y_{c} Y_{t}\right)^{1 / 2} \\
& F_{66}=1 / S^{2}
\end{aligned}
$$

where $X$ and $Y$ are the failure stresses in these directions with subscript $c$ and $t$ meaning "compression" and "tension", and $S$ is the shear failure stress. The stresses on the plate are also calculated with an originally developed FEA code with 8-node iso-parametric elements. 


\section{Numerical results and discussions}

\subsection{Calculation conditions}

Numerical results here are calculated with elastic constants for a graphite/epoxy (CFRP) composite: $E_{1}=138 \mathrm{GPa}, E_{2}=8.96 \mathrm{GPa}, G_{12}=7.10 \mathrm{GPa}$, and $v_{12}=0.3$ with the failure stresses [21]: $X_{t}=X_{c}=144800 \mathrm{~N} / \mathrm{cm}^{2}, \underline{Y_{t}}=5171 \mathrm{~N} / \mathrm{cm}^{2}, \underline{Y}_{c}=20685 \mathrm{~N} / \mathrm{cm}^{2}$, and $S=9008 \mathrm{~N} / \mathrm{cm}^{2}$. Genetic parameters are follows: $p_{c}=0.9, L=7, N_{g}=400, N_{p}=400$, and $p_{\max }=0.1$, where $N_{g}$ and $N_{p}$ are the numbers of generations and populations. The present study employs the maximum number of generation as termination criteria due to simpleness, and the number of generation was determined by a try and error approach by confirming the convergence of solutions.

\section{$\underline{\text { 3.1.1 Problem (i), natural frequency problem }}$}

Figure 5 shows the boundary conditions of the plates for problem (i). The plates are square with a thickness ratio $a / h=100$, and the mesh is a reticular pattern with 100 elements and 341 nodes: (a) CSFF is the plate with asymmetric boundary conditions, the left edge is clamped, the lower edge is simply supported, and other edges are free; (b) CCCC is a totally clamped plate.

\subsubsection{Problem (ii), in-plane strength problem}

Figure 6 shows the model for the calculation. Due to the symmetry, only a quarter model of the plate with a circular hole is considered under a uni-axial tensile stress, $\sigma_{t}=10 \mathrm{MPa}$. The dimensions of the model are $w=0.1 \mathrm{~m}, a=0.05 \mathrm{~m}, c=0.7 \mathrm{~m}, h$ (thickness) $=0.001 \mathrm{~m}$ assuming a plate with an infinite length but finite width. The horizontal and vertical directions are constrained rigidly on the left and bottom edges (symmetric boundaries). Figure 7 shows the finite element model used in this study, and the numbers of nodes and elements are 1335 and 400. The failure indexes are only evaluated in the region around the circular hole $(0 \leq x \leq 0.1 \mathrm{~m}$, 
$0 \leq y \leq 0.1 \mathrm{~m}$ ) shown as the gray colored area in Fig. 6 since nodes in other area have lower Tsai-Wu indexes than in the vicinity of circular hole. The curvature is only evaluated in the "+layer" in the present optimization since the "-layer" has similar average values of curvatures as the "+layer".

Considering the continuity of curvilinear fibers on the symmetric edges, the following constraint is imposed on the shape coefficients $c_{i j}$.

$$
c_{10}=c_{01}=c_{11}=c_{12}=c_{21}=0
$$

where Eq. (9) includes two constraints (C1) and (C2):

(C1) $c_{10}=c_{11}=c_{12}=\left.0 \Rightarrow \frac{\partial f}{\partial x}\right|_{x=0}=\left.0 \Rightarrow \tan \theta\right|_{x=0}=0 \Rightarrow \theta=0^{\circ}$ on $y$ axis
(C2) $c_{01}=c_{11}=c_{21}=\left.0 \Rightarrow \frac{\partial f}{\partial y}\right|_{y=0}=\left.0 \Rightarrow \tan \theta\right|_{y=0}=0$ or $\pm \infty \Rightarrow \theta=0^{\circ}$ or $90^{\circ}$ on $x$ axis

In constraint (C2), the fiber orientation angle $\theta$ only becomes $0^{\circ}$ for $\partial f / \partial x=0$ at the $x$ axis, and it is $\theta=90^{\circ}$ in other cases. It is possible to impose a different set of constraints, $\theta=0^{\circ}$ or $90^{\circ}$ at the $y$ axis and $\theta=0^{\circ}$ at the $x$ axis, on the fiber shape, but numerical experiments showed that the $\theta=90^{\circ}$ at the $y$ axis fiber shape has disadvantages in terms of in-plane strength in the unidirectional tensile state of the $x$ direction, due to a low failure strength normal to the fiber direction of the fibrous composite. Thus, only the constraint expressed by Eq. (9) is employed here.

\subsection{Pareto-optimum solutions}

\subsubsection{Problem (i), natural frequency problem}

Figure 8 shows the calculated Pareto-optimum solutions for the CSFF plate. The vertical and horizontal axes are the average curvature $\bar{\kappa}$ in Eq. (4) and the frequency parameter $\Omega$ defined in Eq. (6). The number of solutions with rank 1 in the last generation is 132, and they are widely distributed ranging from plates with straight fibers to plates with average curvatures 
of 4.91. Figure 9 illustrates the fiber shapes of the "+ layer" for solutions (a), (b), and (c) in Fig. 8. The lines are contours dividing polynomial surfaces into 30 equal heights, and the spacing is unrelated to the fiber volume fraction which is assumed as constant in each finite element here. The design (c) with the highest frequency parameter has fiber shapes normal to the left clamped edge, and fibers in design (b) incline toward the clamped edge and have $1.5 \%$ lower frequencies than design (a). Design (c) shows almost straight fibers with an average curvature of $8.0 \times 10^{-3}[1 / \mathrm{m}]$.

The results for the totally clamped plate (CCCC) are plotted in Fig. 10 and solutions (d), (e), and (f) are shown in Fig. 11. The number of Pareto-optimum solutions is 370 divided into two groups. One group has large curvatures above 20 and the other includes solutions with curvatures lower than 15. Design (f) has the highest fundamental frequency; the fiber shape is radial and all fibers are oriented normal to the clamped edges. All solutions in the first group have fiber shapes similar to design (f). Discretized fiber shapes of design (f) and details of four center elements are shown in Fig. 12, where fibers in the center elements form closed shapes. It is impossible to fabricate, but the designer can avoid such shapes using the present Pareto-optimum solutions. Design (e) has unbent curves and design (d) shows nearly straight fibers with an average curvature of $5.3 \times 10^{-4}[1 / \mathrm{m}]$. The first group with large curvatures, including design (f), is difficult to produce due to the large curvatures, but design (e) in the group with low curvatures has regularly curving fibers which may be possible to produce. These results show the advantages of the present multi-objective optimization. From the above results, the curvilinear fibers result in higher fundamental frequencies than the straight fibers. The shape coefficients, fundamental frequencies, and curvatures for solutions (a)-(f) are listed in Table 1. 


\subsubsection{Problem (ii), in-plane strength problem}

Figure 13 shows the obtained Pareto-optimum solutions where the vertical and horizontal axes are the average curvature and the Tsai-Wu failure index. Figure 14 shows the curvilinear fiber shapes of the "+layer" corresponding to solutions (g), (h), (i) and (j) indicated in Fig. 13. Designs (g) and (h) show fiber shapes of $\theta=0^{\circ}$ at the $y$ axis and $\theta=90^{\circ}$ at the $x$ axis, and the in-plane strength decreases as the curvature decreases. Design (i) shows the fiber shape for $\theta=$ $0^{\circ}$ on $x$ axis with small curvatures and design (j) has zero curvatures, straight fibers. Both designs (i) and (j) indicate higher failure indexes than the others. This suggests that composite plates reinforced by curved fibers with large curvatures have a higher in-plane strength than plates with straight fibers, showing the trade-off relation between fiber curvature and in-plane strength, and the present method successfully generates results with the widely distributed Pareto-optimum solutions.

Table 2 lists the Tsai-Wu failure indexes, average curvatures, and obtained shape coefficients for the solutions in Fig. 14. Design (j) with straight fibers has the highest Tsai-Wu failure index, and this demonstrates an advantage of curvilinear fibers in terms of the in-plane strength. Figure 15 shows discretized fiber shapes of the +layer, -layer, and overlapping view of the two for design (g) which has the lowest failure index. The fiber orientation in the vicinity of the hole in the-layer shows fiber shapes which are oriented tangentially around the circular hole. This agrees well with the naturally occurring hole in bone referred to in Ref. [2] and substantiates the validity of the present modeling and optimization method. 


\section{Conclusions}

The present study proposes a multi-objective optimization method for fibrous composite plates with curvilinear fibers to maximize the mechanical properties, including fundamental frequencies or in-plane strengths, and minimizing the average curvatures of fibers. Two mechanical properties are assigned as conflicting objectives to the curvatures. The first is the fundamental frequency of the plate, and the second is the in-plane strength represented by the Tsai-Wu failure index. Curvilinear fiber shapes are defined by the projections of contour lines for a cubic polynomial surface, and the coefficients of the polynomial are employed as design variables. An improved non-dominated sorting genetic algorithm (NSGA-II) is used as the optimizer with a mutation where the probability is controlled to maintain variety among individuals and to sustain the search ability. A set of constraints to maintain symmetry of fibers at symmetric boundaries is imposed on the design variables.

The numerical results showed that the present optimization method resulted in a widely distributed Pareto-optimum solutions ranging from straight to much curved fibers. The solutions have higher fundamental frequencies and in-plane strengths than plates with straight fibers, demonstrating an advantage of curvilinear fibers. Curvilinear fiber shapes shows similarities with the natural compounds, and this supports the validity of the present results.

\section{Acknowledgements}

This work was supported by a Grant-in-Aid for Young Scientists B (No. 22760164). 


\section{Reference}

1. Lopes CS, Gürdal Z, Camanho PP, Variable-stiffness composite panels: Buckling and first-ply failure improvements over straight-fibre laminates. Comput Struct 2008; 86(9): 897-907.

2. Giri B, Tadano S, Fujisaki K, Todoh M, Microstructure of bone around natural holen in bovine lumbar vertebra. JBSE 2007; 2(1): 1-11.

3. Honda S, Narita Y, Vibration design of laminated fibrous composite plates with local anisotropy induced by short fibers and curvilinear fibers. Compos Struct 2011; 93(2): 902-910.

4. Martin AF, Leissa AW, Application of the Ritz method to plane elasticity problems for composite sheets with variable fiber spacing. Int J Numer Meth Eeg 1989; 28(8): 1813-1825.

5. Hyer MH, Lee HH, The use of curvilinear fiber format to improve buckling resistance of composite plates with central circular holes. Compos Struct 1991; 18: 239-261.

6. Wu KC, Gürdal Z, Starnes, Jr. JH. Structural response of compression loaded, tow-placed, variable stiffness panels. In: Proceedings of the $43^{\text {rd }}$ AIAA/ASME/ASCE/AHS/ASC structures, structural dynamics and materials conference, AIAA 2002-1512; Denver, CO; 2002.

7. Gürdal Z, Olmedo R, In-plane response of laminates with spatially varying fiber orientation: variable stiffness concept. AIAA J 1993; 31(4): 751-758.

8. Gürdal Z, Tatting BF, Wu CK, Variable stiffness composite panels: effects of stiffness variation on the in-plane and buckling response. Compos Part B: Eng 2008; 39(5): 911-922.

9. Lopes CS, Camanho PP, Gürdal Z, Tatting BF, Progressive failure analysis of tow-placed, variable-stiffness panels. Int J Solids Struct 2007; 44(25-26): 8493-8516.

10. Blom AW, Lopes CS, Kromwijk PJ, Gürdal Z, Camanho P. A theoretical model to study the influence of tow-drop areas on the stiffness and strength of variable stiffness laminates. $\mathrm{J}$ Compos Mater 2009; 43(5): 403-25.

11. Blom AW, Tatting BF, Hol JMAM, Gürdal Z. Fiber path definitions for elastically tailored conical shells. Compos Part B: Eng 2009; 40(1): 77-84.

12. Honda S, Narita Y, Natural Frequencies and vibration modes of laminated composite plates reinforced with arbitrary curvilinear fiber shape paths. J Sound Vib 2012; 331(1): 180-191.

13. Setoodeh S, Abdalla MM, Gürdal Z., Design of variable-stiffness laminates using lamination parameters. Compos Part B: Eng 2006; 37(4-5): 301-309.

14. Abdalla MM, Setoodeh S, Gürdal Z, Design of variable stiffness composite panels for maximum fundamental frequency using lamination parameters. Compos Struct 2007; 81(2): 283-291.

15. IJsselmuiden ST, Abdalla MM, Gürdal Z. Optimization of variable-stiffness panels for maximum buckling load using lamination parameters. AIAA J 2010; 48(1):134-43. 
16. Van Campen, JMJF., Kassapoglou C, Gürdal Z, Generating realistic laminate fiber angle distributions for optimal variable stiffness laminates. Compos Part B: Eng 2012; 43(2): 354 360.

17. Honda S, Narita Y, Design of composite plates with optimally distributed short fibers. In: Proceeding of ICCM-16 Conference. Kyoto, July 2007. CD-ROM (MoKA1-03).

18. Blom AW, Setoodeh S, Hol JMAM, Gürdal Z. Design of variable-stiffness conical shells for maximum fundamental eigenfrequency. Comput Struct 2008; 86(9): 870-878.

19. Blom AW, Abdalla MM, Gürdal Z, Optimization of course locations in fiber placed panels for general fibre angle distributions. Compos Sci Technol 2010; 70(4): 564-70.

20. Cho HK, Rowlands RE, Reducing tensile stress concentration in perforated hybrid laminate by genetic algorithm. Compos Sci Technol 2007; 67(13): 2877-2883.

21. Huang J, Haftka RT, Optimization of fiber orientation near a hole for increased load-carrying capacity of composite. Struct Multidiscip O 2005; 30(5): 335-341.

22. Parnas L, Oral O, Ceyhan Ü, Optimum design of composite structures with curved fiber courses. Compos Sci Technol 2003; 63(7): 1071-1082.

23. Pelletier JL, Senthil VS, Multi-objective Optimization of fiber reinforced composite laminates for strength, stiffness and minimal mass. Comput Struct 2006; 84(29-30); 2065-2080.

24. Lee DS, Morillo C, Bugeda G, Oller S, Onate E, Multilayered composite structure design optimisation using distributed/parallel multi-objective evolutionary algorithms. Comput Struct 2012; 94(3): 1087-1096.

25. Mahmoud MRT, Arife BCA, Marwan AH, A multi-objective optimization approach for design of blast-resistant composite laminates using carbon nanotubes. Compos Part B: Eng 2009; 40(6): 522-529.

26. Deb K, Multi-objective optimization using evolutionary algorithms. New York; John Wiley and Sons Ltd, 2001.

27. Zienkiewicz OC, The finite element method in engineering science 2nd edition, London; McGraw - Hill, 1971.

28. Reddy JN, Mechanics of laminated composite plates theory and analysis, CRC Press, Inc., 1997. 


\section{Captions of figures and Tables}

[Figures]

Fig. 1. Example of surface with corresponding curves and discretized fiber shapes.

Fig. 2. Schematic representation of the non-dominated sorting for two objectives.

Fig. 3. Schematic representation of crowded distance metric for two objectives.

Fig. 4. Flow chart of NSGA-II.

Fig. 5. Boundary conditions for (a) plate with asymmetric boundaries (CSFF) and (b) totally clamped (CCCC) plate for problem (i).

Fig. 6. Dimensions and boundary conditions for problem (ii).

Fig. 7. Finite element model for problem (ii).

Fig. 8. Pareto-optimum solutions of CSFF plate for problem (i).

Fig. 9. Representative fiber shapes (a), (b), and (c) for CSFF plate.

Fig. 10. Pareto-optimum solutions of CCCC plate for problem (i).

Fig. 11. Representative fiber shapes (d), (e), and (f) for CCCC plate.

Fig. 12. Discretized fiber shapes for design (f) and details of four center elements.

Fig. 13. Pareto-optimum solutions for problem (ii).

Fig. 14. Representative fiber shapes (g), (h), (i), and (j) for problem (ii).

Fig. 15. Discretized fiber shapes for design (g), “+layer”, “-layer”, and overlapping of the two.

[Tables]

Table 1. Shape coefficients, frequency parameters, and average curvatures of designs (a) - (f) for problem (i).

Table 2. Shape coefficients, Tsai-Wu failure indexes, and average curvatures of designs (g) - (j) for problem (ii). 


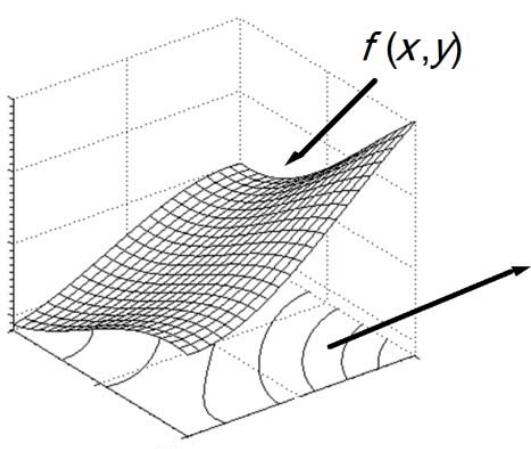

(a) surface

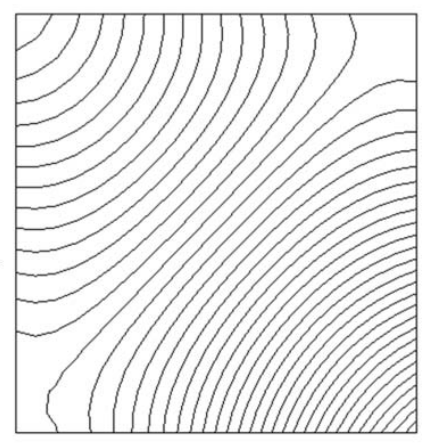

(b) continuous

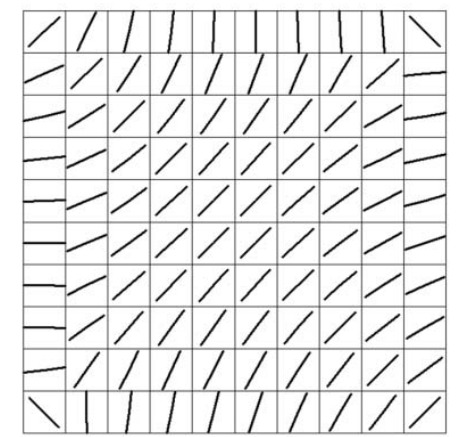

(c) discrete

Figure 1.

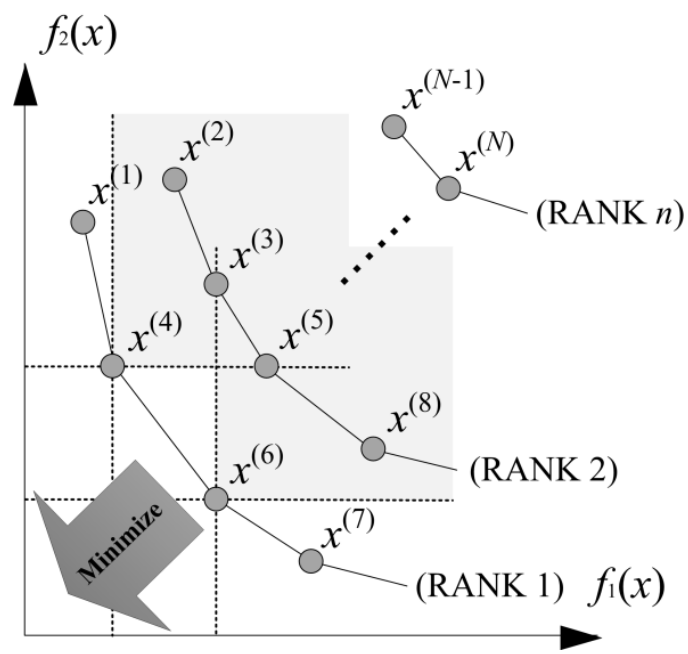

Figure 2.

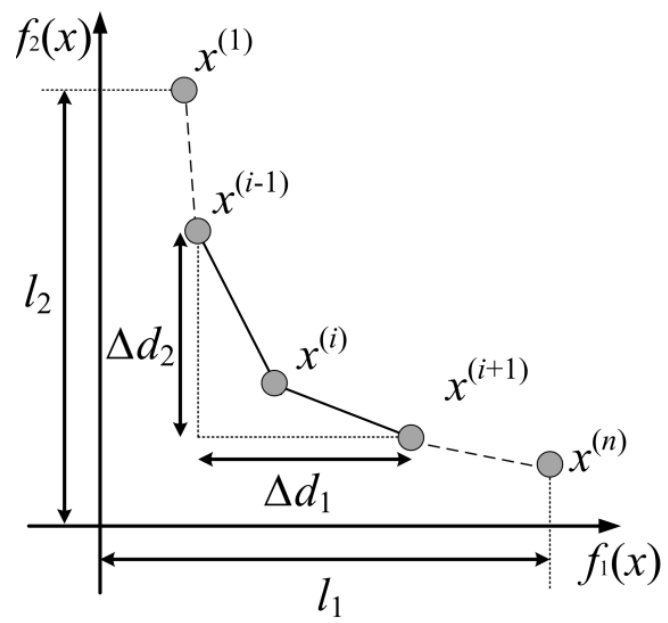

Figure 3. 


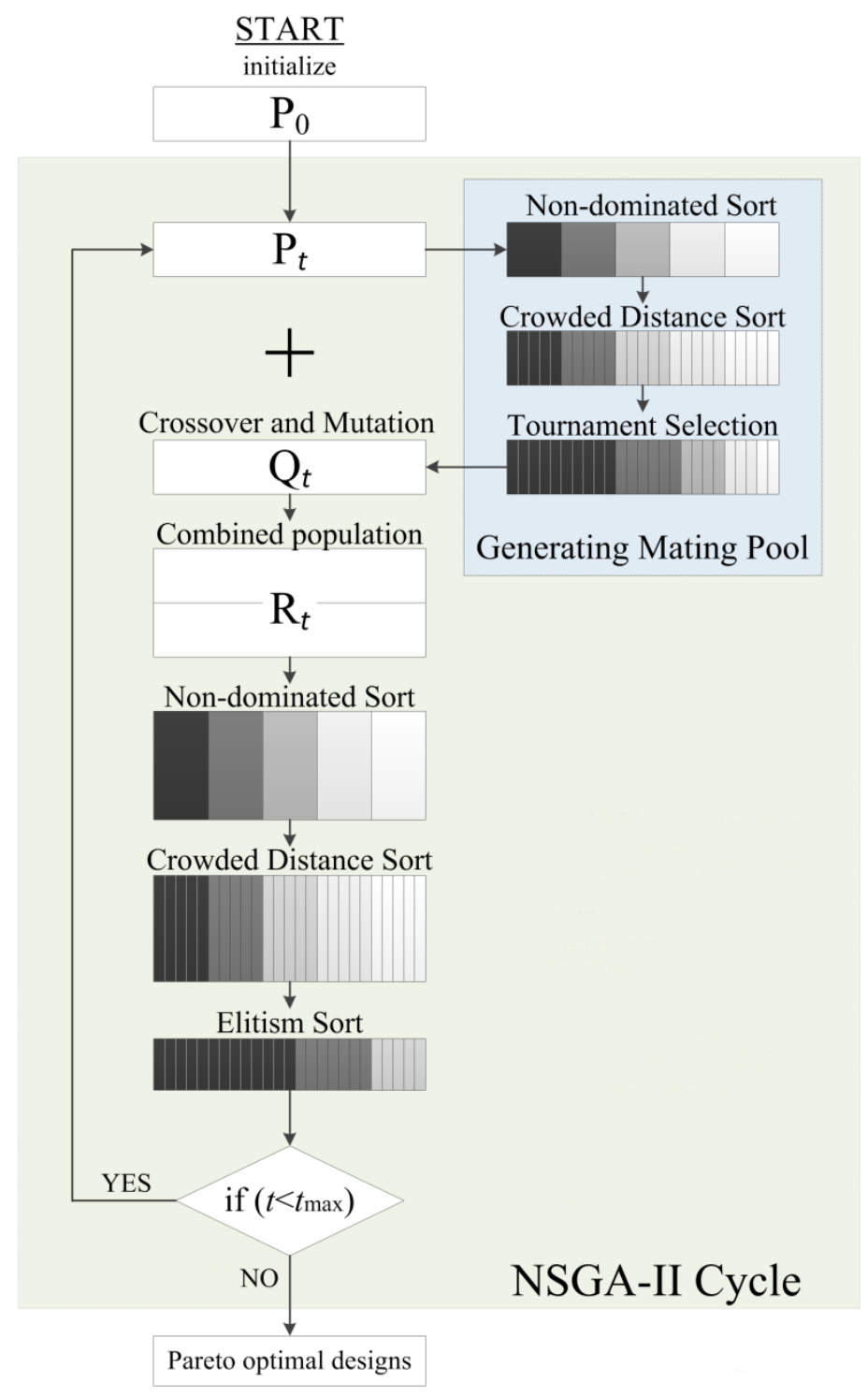

Figure 4.

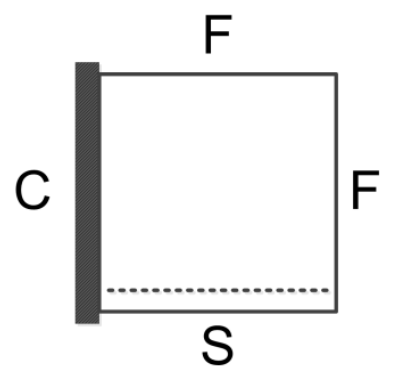

(a)CSFF

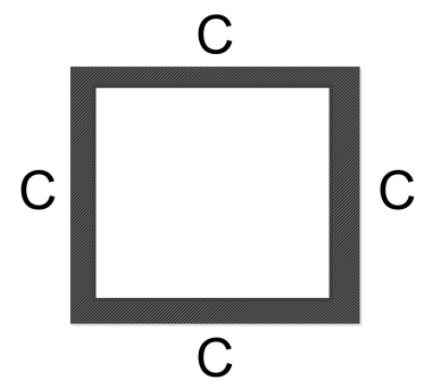

(b) CCCC

Figure 5. 


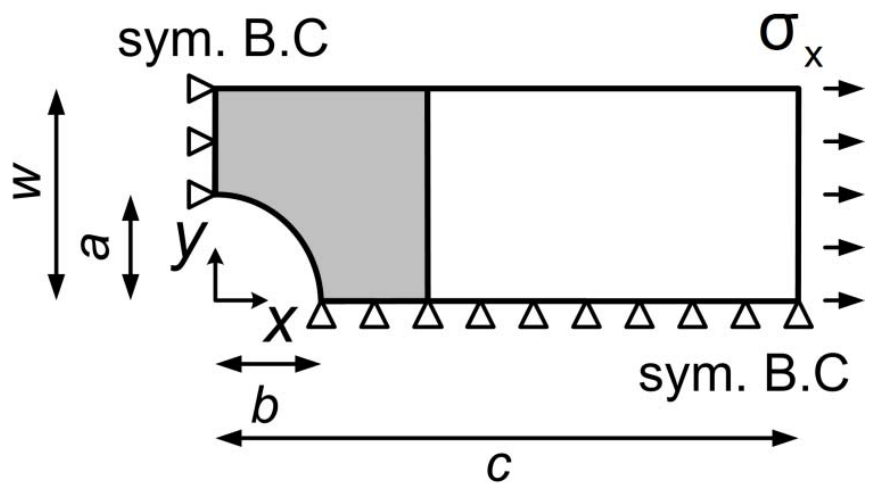

Figure 6.

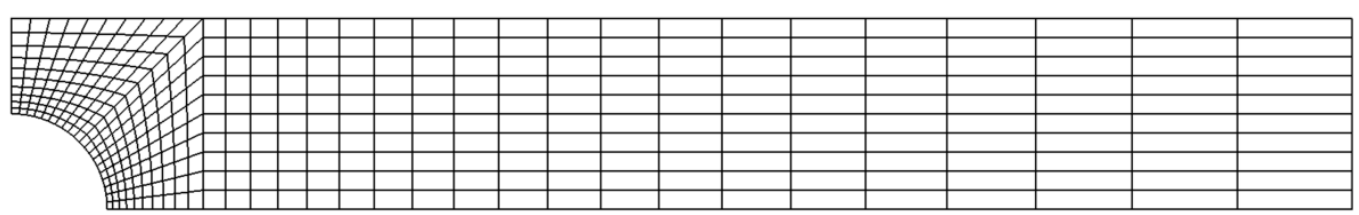

Figure 7.

CSFF

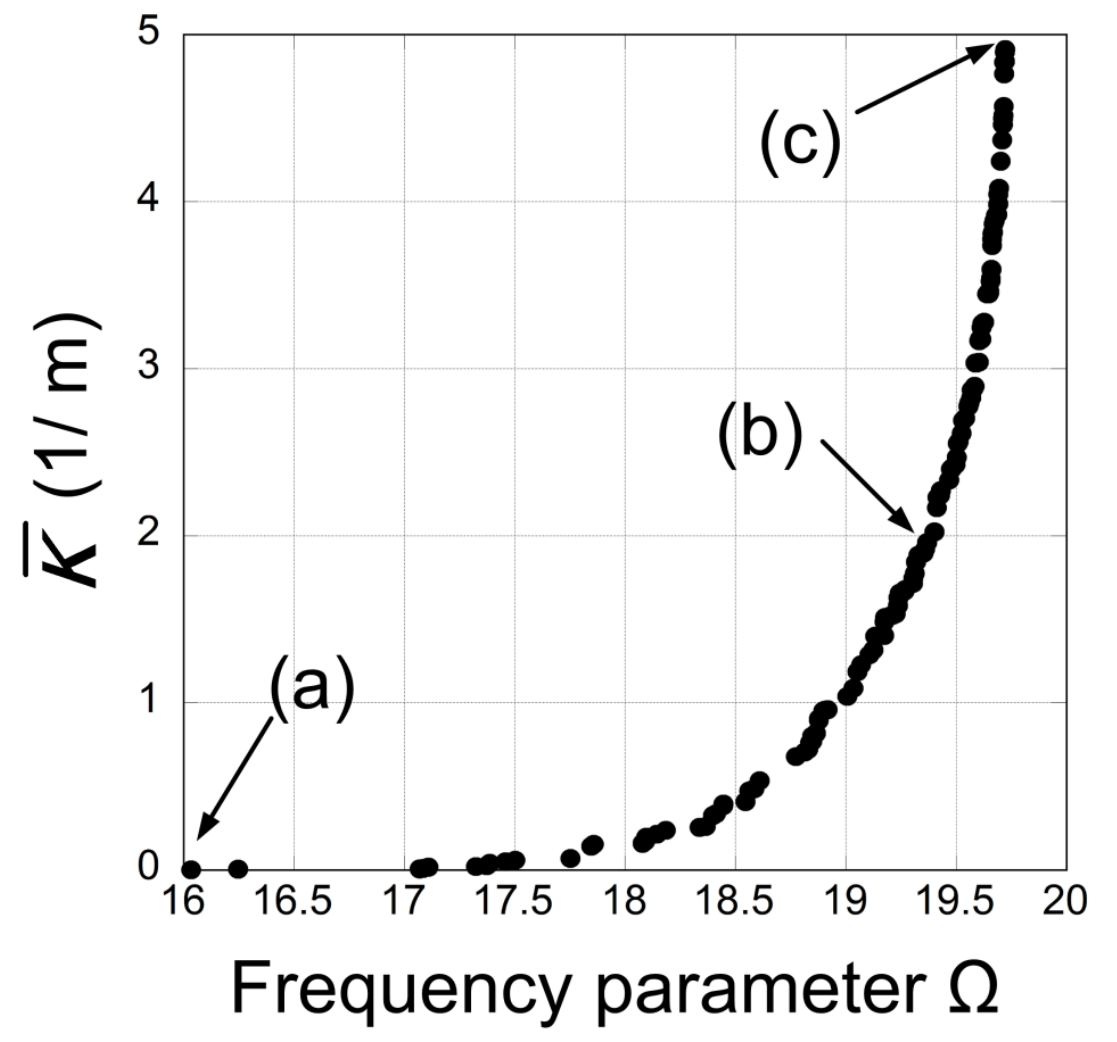

Figure 8. 


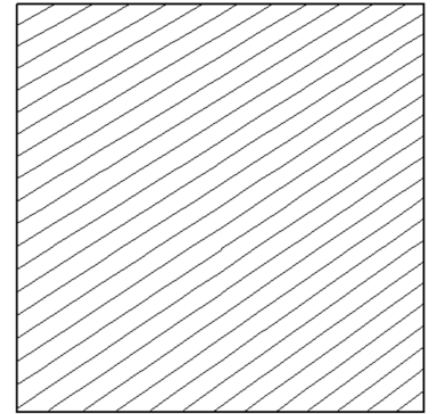

Design (a)

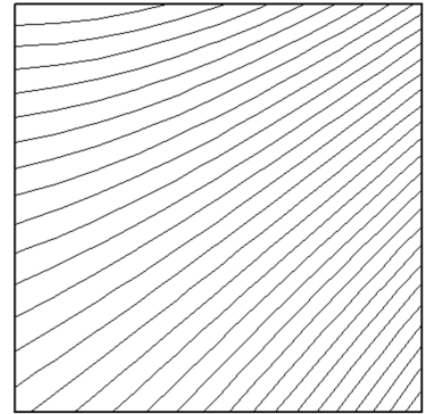

Design (b)

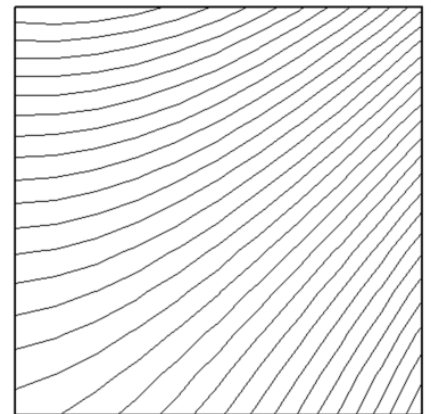

Design (c)

Figure 9.

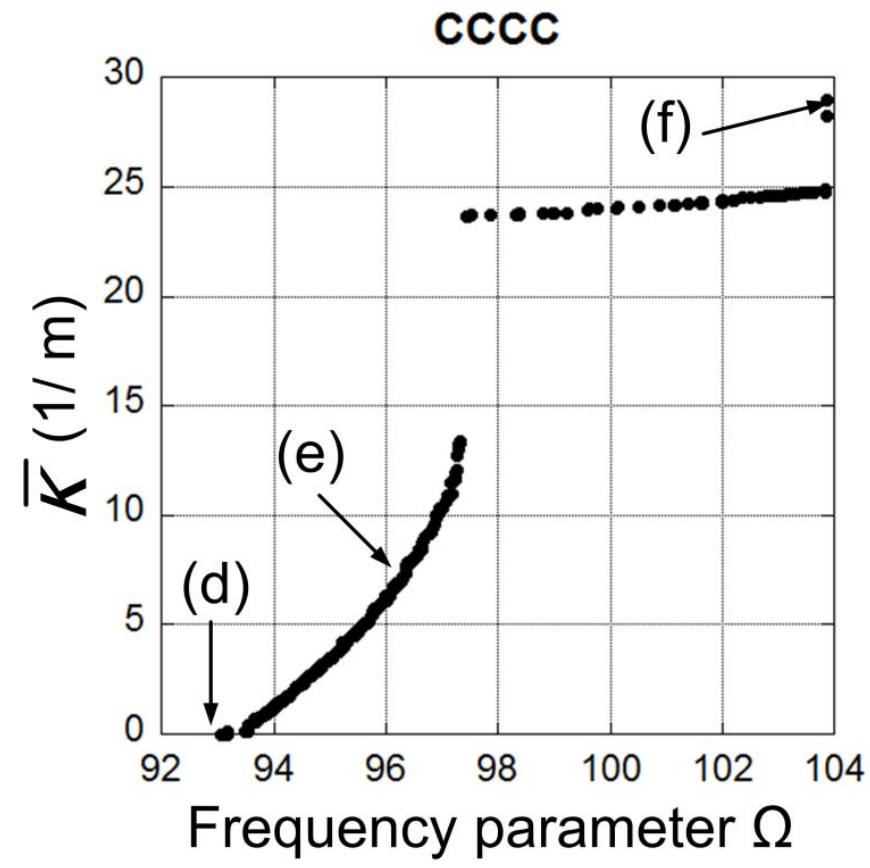

Figure 10.

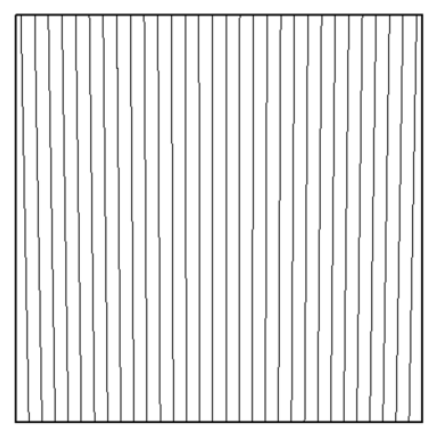

Design (d)

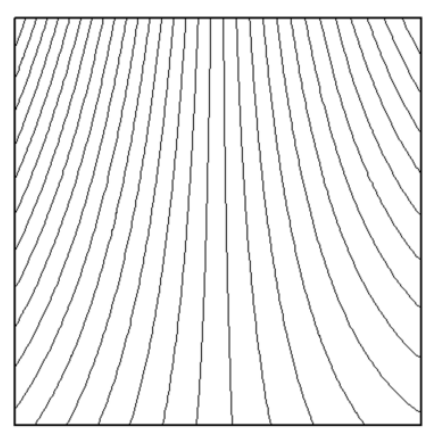

Design (e)

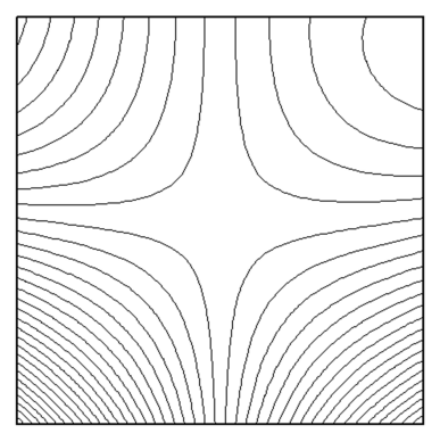

Design (f)

Figure 11. 


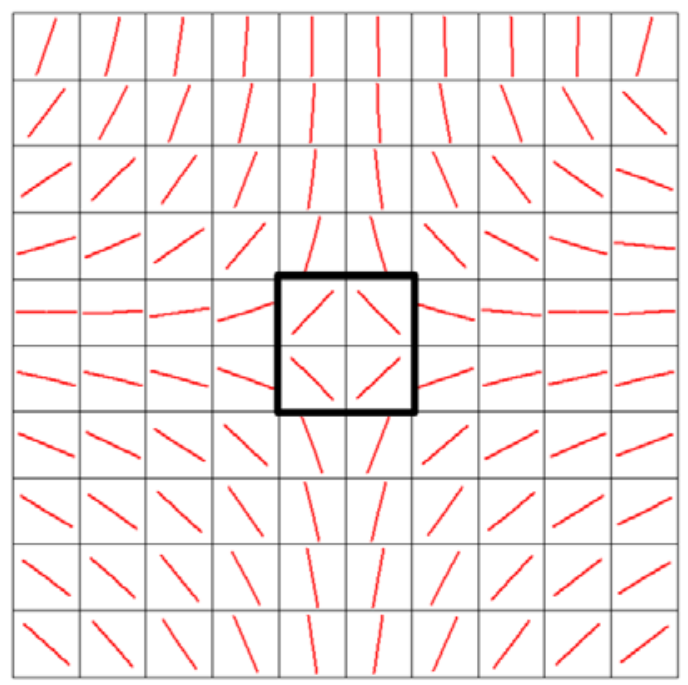

Descretized fiber shape of Design (f)

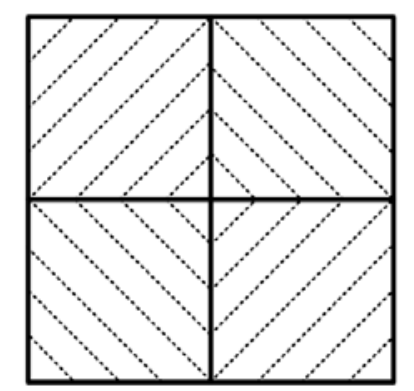

Details of four center elements

Figure 12.

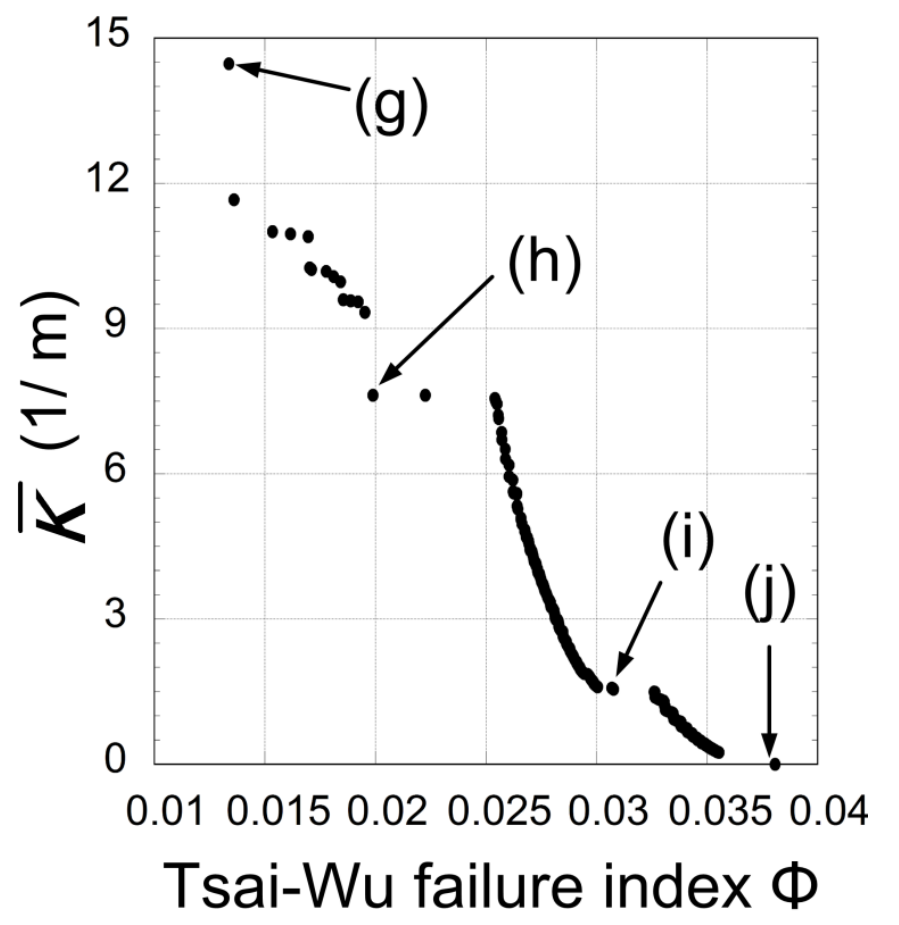

Figure 13. 


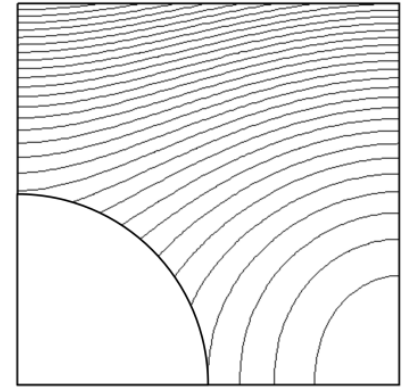

Design (g)

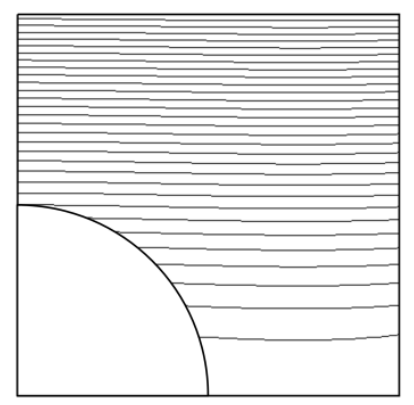

Design (i)

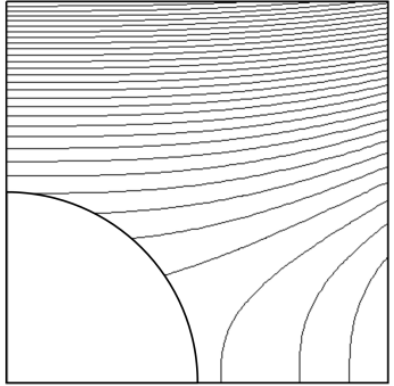

Design (h)

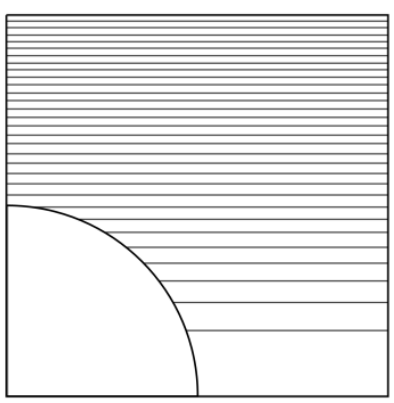

Design (j)

Figure 14.

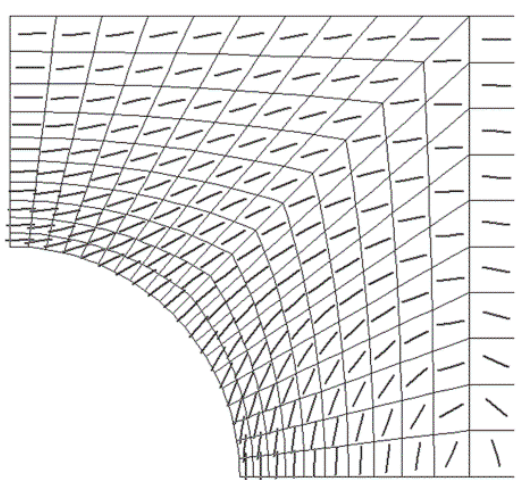

+ layer

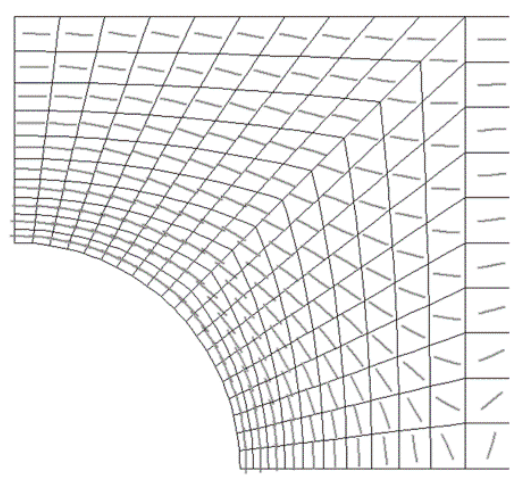

- layer

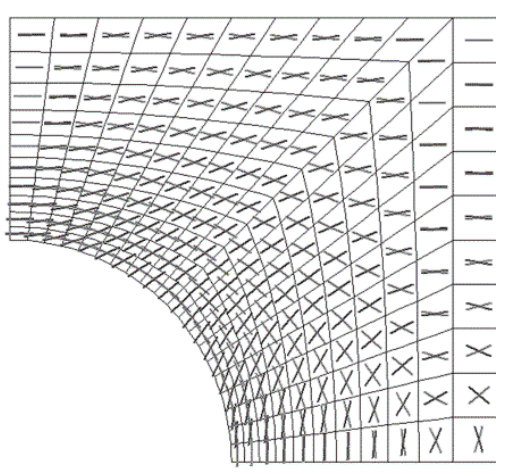

Overlapping

Figure 15. 
Table 1

\begin{tabular}{cccccccccccc}
\hline \multirow{2}{*}{ Designs } & \multicolumn{1}{c}{ The optimized shape coefficients } & & \multicolumn{1}{c}{$\begin{array}{c}\text { Frequency } \\
\text { parameters }\end{array} \begin{array}{c}\text { Average } \\
\text { curvatures }\end{array}$} \\
\cline { 2 - 13 } & $c_{10}$ & $c_{01}$ & $c_{20}$ & $c_{11}$ & $c_{02}$ & $c_{30}$ & $c_{21}$ & $c_{12}$ & $c_{03}$ & $\Omega$ & $\bar{\kappa}(1 / \mathrm{m})$ \\
\hline $\mathrm{a}$ & -1.3 & 2.0 & -1.3 & 1.4 & 0.9 & -1.5 & 1.9 & -0.4 & 1.8 & 17.13 & $8.0 \times 10^{-3}$ \\
$\mathrm{~b}$ & -0.2 & 0.3 & -1.5 & 1.3 & 0.8 & -1.9 & -0.4 & 1.7 & 1.8 & 19.41 & 2.09 \\
$\mathrm{c}$ & -0.2 & 0.3 & -2.0 & 0.6 & 1.3 & -0.9 & -2.0 & 0.6 & -0.8 & 19.72 & 4.91 \\
\hline $\mathrm{d}$ & -2.0 & 0.0 & -0.1 & 0.8 & 0.0 & -0.9 & -0.3 & -0.3 & 0.0 & 93.08 & $5.3 \times 10^{-4}$ \\
$\mathrm{e}$ & 0.1 & 0.0 & -0.3 & 1.0 & 0.0 & -1.7 & -2.0 & 2.0 & -0.1 & 95.64 & 5.06 \\
$\mathrm{f}$ & 0.0 & 0.0 & 0.0 & -0.2 & 0.0 & 0.2 & 0.6 & 2.0 & 0.0 & 103.9 & 29.03 \\
\hline
\end{tabular}

Table 2

\begin{tabular}{ccccccc}
\hline \multirow{2}{*}{ Designs } & \multicolumn{3}{c}{$\begin{array}{c}\text { The optimized shape } \\
\text { coefficients }\end{array}$} & $\begin{array}{c}\text { Tsai-Wu } \\
\text { Failure index }\end{array}$ & $\begin{array}{c}\text { Average } \\
\text { curvatures }\end{array}$ \\
\cline { 2 - 7 } & $c_{20}$ & $c_{02}$ & $c_{30}$ & $c_{03}$ & $\Phi$ & $\bar{\kappa}(1 / \mathrm{m})$ \\
\hline $\mathrm{g}$ & -0.3 & 0.1 & 2 & 1.9 & 0.0134 & 14.48 \\
$\mathrm{~h}$ & 0 & 0 & -0.2 & 1.5 & 0.0199 & 7.630 \\
$\mathrm{i}$ & 0.1 & 2 & -0.9 & 2 & 0.0308 & 1.552 \\
$\mathrm{j}$ & 0 & 1.8 & 0 & -1.5 & 0.0381 & 0 \\
\hline
\end{tabular}

